\title{
Tungsten behaviour in radiatively cooled plasma discharges in ASDEX Upgrade
}

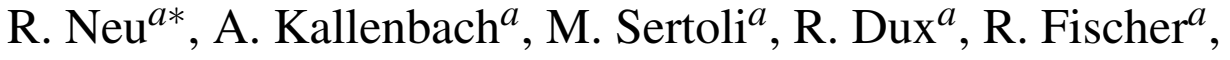 \\ J.C. Fuchs ${ }^{a}$, A. Janzer ${ }^{a}$, H.W. Müller ${ }^{a}$, S. Potzel ${ }^{a}$, T. Pütterich ${ }^{a}$, \\ G. van Rooij ${ }^{b}$, ASDEX Upgrade Team ${ }^{a}$ \\ ${ }^{a}$ Max-Planck-Institut für Plasmaphysik, EURATOM-Association, D-85748 Garching, \\ Germany \\ ${ }^{b}$ FOM-Institute for Plasma Physics Rijnhuizen, Assoc. Euratom-FOM, Trilateral Euregio \\ Cluster, 3430 BE, Nieuwegein, The Netherlands
}

\begin{abstract}
In the all W ASDEX Upgrade impurities are injected routinely for radiative cooling. Amongst the different species used, nitrogen has been shown to provide the best cooling effect, while maintaining or even improving the energy confinement. Depending on the divertor plasma parameters a reduction or increase of the $\mathrm{W}$ sputtering yield compared to the unseeded phases is observed. Although usually the energy confinement is improved during $\mathrm{N}_{2}$ seeding, no increased W concentrations are observed. Obviously the particle confinement is not improved and there are no signs of central density peaking. Moreover the sustained regular ELM activity prevents a strong inward transport of $\mathrm{W}$ across the pedestal region. In contrast, when using Ar as seeding impurity the ELM frequency is strongly reduced and also a change of central particle transport is observed provoking peaking of the $\mathrm{W}$ density profile.
\end{abstract}

PSI-19 keywords:

Tungsten, Impurity transport, Impurity sources, Radiation, ASDEX Upgrade 
JNM keywords:

P0500, I0100, P0600, S1300, T1000

PACS numbers:

52.25.Vy, 52.40.Hf, 52.55.Rk, 52.55.Fa

* Corresponding author address: Boltzmannstr.2 85748 Garching

* Corresponding author E-mail: Rudolf.Neu@ipp.mpg.de

Presenting author: Rudolf Neu

Presenting author e-mail: Rudolf.Neu@ipp.mpg.de

\section{Introduction}

Reduction of the concentrated power load in the divertor will be mandatory for future fusion devices, independent of the choice of plasma facing material (PFM) used. Moreover, abandoning carbon based plasma facing components (PFCs) will aggravate the issue because its ability to radiate efficiently at plasma temperatures ambient in the divertor will be absent. ASDEX Upgrade, which has been operated with all tungsten (W) PFCs since 2007 [1], faces a similar situation, after the C and $\mathrm{O}$ concentrations were reduced to $\approx 0.1 \%$ by boronisation [2]. As a consequence, the power loads in the divertor can easily exceed $10 \mathrm{MWm}^{-2}$ and endanger the W coatings used in AUG. Therefore, impurities are injected routinely for radiative cooling. Amongst the different species used, nitrogen has been shown to provide the best cooling effect in ASDEX Upgrade discharges, while maintaining or even improving the energy confinement $[3,4]$. This paper presents a characterisation of the $\mathrm{W}$ erosion in impurity seeded discharges depending on divertor temperature and impurity puff rate, which are varied independently. In addition, the behaviour of the divertor erosion yield as a function of the $\mathrm{N}$ flux is investigated (see Sec. 3). Although the $\mathrm{W}$ influx is a necessary prerequisite for the central $\mathrm{W}$ concentration 
$\left(c_{W}\right)$, it has been shown that $c_{W}$ is mainly governed by transport [5,6]. Since the energy confinement increases regularly in $\mathrm{N}_{2}$ seeded discharges the question arises whether this is also true for the $\mathrm{W}$ content. The behaviour of the $\mathrm{W}$ concentration in impurity seeded discharges will be described in more detail in Sec. 4. There the influence of gas mixtures on the discharge behaviour will also be investigated. Finally Sec. 5 concludes the paper.

\section{Impurity seeded discharges in ASDEX Upgrade}

In order to provide a flexible and optimized protection of the divertor from excessive heat loads, a feedback signal is required which is closely related to the target power load. There are several approaches, but their complexity is critical to a system relevant for machine safety. In ASDEX Upgrade, the ELM filtered (thermo-) electric current into a divertor tile, measured as voltage at a shunt resistor embedded in the tile mounting has been chosen as a feedback signal. This measurement probes mainly the temperature difference between the outer and inner divertor. The inner divertor has very low electron temperatures in between ELMs and therefore the thermoelectric current corresponds in good approximation to the outer divertor temperature as could be confirmed by Langmuir probe measurements. Since the interpretation of the measured signal in terms of a divertor temperature appeared more useful for operation and interpretation, the current is multiplied by a fixed factor obtained by comparison with Langmuir probe data and termed $T_{e}^{d i v}$ throughout this paper, with units of electron volts. Within this unit the error of the measurement is estimated to be smaller than $2 \mathrm{eV}$. For H-mode conditions, $T_{e}^{d i v}$ was found to also represent a reasonable approximation of the outer divertor peak power load [7]. It turned out to be very simple and robust in real-time data acquisition and evaluation. In first attempts performed with argon seeding a considerable fraction of central heating had to be used to avoid central impurity peaking. More- 
over, since the radiative power removal by Ar takes place in the separatrix/pedestal region, the discharges were close to the HL threshold causing large, low frequency type-I ELMs leading in turn to a strong increase of the bulk impurity level and radiation. Active ELM triggering by injection of small pellets was necessary to allow for steady operation [8]. In contrast using the same control scheme but $\mathrm{N}_{2}$ instead of $\mathrm{Ar}$ as seeding gas, a very beneficial behaviour in terms of power load as well as impurity control could be achieved. However, wall storage of nitrogen in tungsten was found to have a significant influence on the necessary gas fluxes and on the nitrogen content of subsequent discharges making the feedback system indispensable [7]. Its qualitative behaviour, namely the $\mathrm{N}$ storage in the implantation range and the nitrogen release at elevated temperature, was found to be similar to that obtained in laboratory experiments [9]. In [4], the radiation characteristic of potential cooling gases is investigated taking into account the non-coronal properties of the edge plasma. It is found that under conditions existent in AUG nitrogen is the most efficient species. Ar is a candidate for radiation cooling in cases of higher edge temperatures, but $\mathrm{Ne}$ seems to be less suited compared to $\mathrm{N}_{2}$ and Ar under most edge plasma conditions.

Typical parameters of radiatively cooled discharges in AUG are $I_{P}=1-1.2 \mathrm{MA}$, which provides a current areal density and therefor a Greenwald density similar to ITER, and $B_{t} \approx 2.5 \mathrm{~T}$ suitable for the resonance frequency of 2 nd harmonic ECRH at $140 \mathrm{GHz}$, resulting in $q_{95}=3.9-4.8$. The heating is varied from $P_{\text {heat }} \approx 6-20$ MW, representing at its upper end a $P / R$ half that of ITER. The divertor temperatures are typically controlled to $T_{e}^{d i v}=4-12 \mathrm{eV}$ resulting in D puffing rates $0.4-4 \cdot 10^{22} \mathrm{e} \mathrm{s}^{-1}$ and $\mathrm{N}_{2}$ puffing rates $0.3-2.8 \cdot 10^{22} \mathrm{e} \mathrm{s}^{-1}$ (the rates are averaged over $200 \mathrm{~ms}$ and account for all electrons of the puffed particles). The $\mathrm{D}_{2}$ was puffed in the outer midplane from 2-4 valves at toroidally different locations, whereas the $\mathrm{N}_{2}$ was injected in the divertor below the X-point toroidally symmetric by 8 outlets. The achieved H-mode scaling factors $H 98 y, 2$ were typically $0.9-1.1$ in unseeded cases,depending mainly on the D puff level, and $H=1.0-1.3$ in seeded cases $[3,4]$. Figure 1 shows a typical $\mathrm{N}_{2}$ seeded discharge with $T_{e}^{\text {div }}$ controlled to 
$8 \mathrm{eV}$. As can be seen in the inserts of the right column the power load to the outer divertor $\left(P_{\text {out }}^{\text {div }}\right)$ is efficiently controlled by the feedback of $T_{e}^{d i v}$.

\section{Behaviour of $W$ influx in impurity seeded discharges}

The time resolved W influx is obtained spectroscopically by measuring the WI line radiation at $400.9 \mathrm{~nm}$ on 38 lines-of-sight covering the outboard divertor, some low field side limiters as well as the central column [10]. The measured photon flux density can be transformed into an eroded tungsten flux density using the inverse photon efficiency, i.e. the $S / X B \approx 20$ (at $T_{e}^{d i v} \approx 10 \mathrm{eV}$ ) [11]. For lower divertor temperatures $\left(T_{e}^{d i v}<5 \mathrm{eV}\right)$ the evaluation of the $\mathrm{W}$ flux gets more uncertain because of the strong temperature dependence of the related $S / X B$ value [12]. One has to keep in mind that the spectroscopically derived W flux only yields an upper limit for the net influx / net erosion since under high density divertor conditions the eroded $\mathrm{W}$ atom gets quickly ionized and may be promptly redeposited within one gyro-orbit. Typical redeposition fractions in the divertor are $50-90 \%[13,14]$.

Comparing a pair of unseeded and $\mathrm{N}_{2}$-seeded discharges with the same heating power and the same level of $\mathrm{D}_{2}$ puff, no difference in the time averaged $\mathrm{W}$ influx was found, neither in the main chamber at the low field side limiters nor in the divertor [7]. Since the radiation cooling by $\mathrm{N}$ is mostly provided in the divertor volume, little change in the main chamber SOL parameters is expected. However in the divertor the inter-ELM temperature decreased from about $12 \mathrm{eV}$ in the unseeded discharges to the feedback controlled to $T_{e}^{d i v}=7 \mathrm{eV}$ in the seeded discharge. Looking into more detail, the influence of $\mathrm{N}$ on the erosion during ELMs was investigated in a discharge series with about $11 \mathrm{MW}$ auxiliary heating and a deuterium puff level of $0.8 \times 10^{22} \mathrm{~s}^{-1}$. The inter-ELM divertor temperatures were feedback controlled by nitrogen puffing to $T_{e}^{d i v}=12,8$ and $5 \mathrm{eV}$ (\#25390-\#25392), respectively. In discharge \#25391 $\left(T_{e}^{d i v}=8 \mathrm{eV}\right)$ the ELM frequency was about a factor 2 higher and the ELMs were a factor 3 shorter compared to \#25390 $\left(T_{e}^{d i v}=12 \mathrm{eV}\right)$. 
Concomitantly, the ELM energies were reduced from $W_{E L M}=60 \mathrm{~kJ}$ (\#25390) to 20 $\mathrm{kJ}$ (\#25391) whereas the requested $\mathrm{N}$ puffing rate increased from $\Gamma_{N} \approx 0.3 \times 10^{22}$ $\mathrm{e} \mathrm{s}^{-1}$ to $\Gamma_{N} \approx 1.4 \times 10^{22} \mathrm{e} \mathrm{s}^{-1}$. Coherent averaging of the fast $\mathrm{W}$ influx measurements reveals that although the ELM energy is only one third of that with less $\mathrm{N}_{2}$ cooling, the maximum $\mathrm{W}$ sputtering yield is 3 times larger during the ELM, leading only to a moderate reduction of the average $\mathrm{W}$ yield [4]. Obviously, the additional sputtering by $\mathrm{N}$ ions compensates for the lower $T_{e}^{d i v}$. Reducing the inter-ELM divertor temperature further to $T_{e}^{d i v}=5 \mathrm{eV}$ (\#25392) leads to an almost complete suppression of inter-ELM W-sputtering in line with TRIM calculations of the effective sputter yield using a few percent of $\mathrm{N}^{3+}$ impinging the $\mathrm{W}$ divertor targets [9].

The behaviour of the $\mathrm{W}$ divertor source, which is by far the largest erosion source [10], is further investigated in Fig. 2. There, the ELM averaged W influx is presented for $\mathrm{H}$-mode discharges at different input power and varying $T_{e}^{d i v}$ or $\mathrm{N}_{2}$ injection, respectively. As can be seen from Fig. 2a) the $\mathrm{W}$ influx in the divertor increases with increasing $P_{a u x}$ (energy and particle flux to the divertor). There is, however, no clear trend for a reduction of the $\mathrm{W}$ influx with decreasing $T_{e}^{d i v}$. The data points with the $\mathrm{W}$ influx close to zero represent discharges where in addition to the $\mathrm{N}_{2}$ puff the $\mathrm{D}_{2}$ puffing rate is increased to a very high level $\left(\Gamma_{D}=4 \times 10^{22}\right.$ $\mathrm{e} \mathrm{s}^{-1}$ ), whereas the other discharges were performed with $\Gamma_{D}=0.4-1.2 \times 10^{22}$ $\mathrm{e} \mathrm{s}^{-1}$. In the latter case the $\mathrm{W}$ influx is extinguished only inter-ELM at $T_{e}^{d i v}<5 e V$, while it is completely suppressed also during the small ELMs (Type III ELMs) appearing at the high $\mathrm{D}_{2}$ puffing level. A quantitative evaluation of the $\mathrm{W}$ influx / yield by spectroscopic means in these cases is however difficult because the strongly decreasing inverse photon efficiency $S / X B$ below $T_{e}=5 \mathrm{eV}$. The same data set is presented in Fig. 2b), but this time plotted against the intensity of a N IV spectral line (at $76.5 \mathrm{~nm}$ ) measured in the main chamber. Again the $\mathrm{W}$ influx is similar for similar levels of $P_{\text {aux }}$ but rather independent from the $\mathrm{N}$ influx. Note that this spectral line has been chosen instead the $\mathrm{N}_{2}$ puffing rate or a $\mathrm{N}$ spectral line from the divertor in order to be less sensitive to variations in $T_{e}^{d i v}$ and to be independent from 
the $\mathrm{N}$ wall storage level. All of the presented discharges are performed in a similar plasma shape and at similar D puffing levels (except the ones at $\Gamma_{D}=4 \times 10^{22}$ $\mathrm{es}^{-1}$ ). Therefore the $\mathrm{N}$ divertor compression is assumed to be similar for all $\mathrm{N}$ levels allowing using the main chamber $\mathrm{N}$ flux as a measure for the divertor fluxes.

\section{Behaviour of $W$ concentration in impurity seeded discharges}

The $\mathrm{W}$ concentration is deduced routinely in AUG from X-ray and VUV spectroscopy [15]. The use of spectral lines origination from charge stages around 25+ as well as around $46+$ allows to distinguish peripheral $\left(T_{e} \approx 1 \mathrm{keV}\right)$ and central emissions $T_{e} \approx 3 \mathrm{keV}$ ) for typical ASDEX Upgrade temperature profiles.

Generally, there is no increase of the $\mathrm{W}$ concentration in $\mathrm{N}_{2}$ seeded discharges compared to non seeded ones (see also [2]). This is exemplified in Fig. 1, where due to the feedback controlled divertor temperature the injection of nitrogen increases by a factor of more than 10 between $\mathrm{t}=2.8 \mathrm{~s}$ and $3.5 \mathrm{~s}$ and the $\mathrm{W}$ concentration remains constant. Moreover, there is no sign of $\mathrm{W}$ accumulation since $c_{W}^{\text {centre }}$ is only slightly higher than $c_{W}^{e d g e}$ throughout the whole discharge. This is true for all discharges with $\mathrm{N}_{2}$ seeding provided there is central ECRH in the range of $10 \%$ of the total auxiliary heating power. This is shown in Fig. 3 where the $\mathrm{W}$ peaking $\left(c_{W}^{\text {centre }} / c_{W}^{\text {edge }}\right)$ as well as $c_{W}^{\text {edge }}$ are plotted against the $\mathrm{N}$ influx. Again the whole data set at different auxiliary heating power $\left(P_{\text {heat }}=6-14 \mathrm{MW}\right)$ and D puffing rates from $0.4-4 \times 10^{22} \mathrm{e} \mathrm{s}^{-1}$ is presented. Typically the line averaged $Z_{e f f}$ increases by $0.5-0.7(1.5-2 \% \mathrm{~N})$ in strongly $\mathrm{N}_{2}$ puffed discharges. The $Z_{e f f}$ profile it is found to be strongly hollow, leading to central $Z_{\text {eff }}$ clearly below 2 . The edge $\mathrm{W}$ concentrations are always in the range of (a few) $10^{-5}$ and the peaking is about two, although the $\mathrm{N}$ influx varies by a factor of 10 . This observation is consistent with the earlier results that $c_{W}^{e d g e}$ is dominated by the main chamber sources [10] - which barely change - provided that the $\mathrm{W}$ transport is invariant. The remaining variation in $c_{W}^{e d g e}$ and the $\mathrm{W}$ peaking is clearly not correlated with the $\mathrm{N}$ influx and 
is mostly attributed to (small) changes in transport caused by different levels of D puffing rates, MHD activity and detailed heating scheme.

The situation changes when using a mixture of $\mathrm{N}_{2}$ and Ar for seeding. Technically, the mixture is provided by feed back controlled $\mathrm{N}_{2}$ injection on which a feedforward Ar puff is overlaid. Fig. 4 shows a similar discharge to the one shown in Fig. 1, where only the heating phase with 7.5 MW from NBI is advanced by $1 \mathrm{~s}$ and $P_{E C R H} \approx 1.5 \mathrm{MW}$ instead of 0.8 MW. As soon as Ar is injected the feedback stops the $\mathrm{N}_{2}$ injection because the divertor temperature is reduced sufficiently by the Ar radiation. After the further increase of the heating power the $\mathrm{N}_{2}$ puff is switched on to control $T_{e}^{d i v}$ again. The Ar puff is programmed in such a way that the central Ar concentration, which is provided by the absolutely calibrated measurement of X-ray radiation emitted from He-like Ar, stays about constant throughout the discharge, as can be seen from the lower left insert. Similar to the discharge with pure $\mathrm{N}_{2}$ injection, the edge $\mathrm{W}$ concentration remains unchanged throughout the discharge. However, as soon as the Ar puff replaces the $\mathrm{N}_{2}$ puff, the central W concentration starts to rise leading to a maximum peaking of $c_{W}^{\text {centre }} / c_{W}^{e d g e}=3$. When the $\mathrm{N}_{2}$ puff starts again the peaking decreases quickly again, albeit the Ar puff and the central Ar concentration remain almost unchanged. The strong influence of the Ar injection on the particle confinement is also reflected in the behaviour of the electron density, which immediately rises and starts to peak (not shown in the figure) although the number of injected electrons is much smaller during this phase (the increase of the Ar and W concentration cannot account alone for the increase of the electron density). During the Ar puff the central $Z_{e f f}$ increases slightly compared to the case with $\mathrm{N}_{2}$ puff only and the $Z_{e f f}$-profile flattens. Part of this behaviour can be explained by the change in the ELM behaviour. Similar to the earlier experiments with Ar puffing [8] the ELMs are less frequent and larger in the Ar dominated phase ( $f_{E L M} \approx 120 \mathrm{~Hz}$ at $3.5 \mathrm{~s}$, instead of $220 \mathrm{~Hz}$ in $\mathrm{N}_{2}$ seeded reference case). An analysis of the particle transport using the behaviour of the spectral line of He-like Ar and soft X-ray profiles employing the gradient-flux method (similar to the procedure described in [16]) indeed shows a change in central transport parameters 
from the first phase at around 2.1-2.3 s, where $\mathrm{N}_{2}$ is puffed in addition, to the phase around 2.5 - 3 s. During the later phase with Ar seeding only, the central diffusion coefficient for Ar is reduced by at least a factor of 3 down to approximately the neoclassical value.

\section{Conclusion and Outlook}

In the all W ASDEX Upgrade impurities are injected routinely for radiative cooling. Amongst the different species used, nitrogen has been shown to provide the best cooling effect in ASDEX Upgrade discharges, while maintaining or even improving the energy confinement. Depending on the background plasma density and the set divertor temperature a reduction or increase of the $\mathrm{W}$ sputtering yield compared to the unseeded phases was observed. An almost complete extinction of the W I intensity can be obtained by a simultaneous increase of the $\mathrm{D}$ and the $\mathrm{N}_{2}$ puffing rate. The increased $\mathrm{D}$ puffing rate can also lead to a transition to the type III ELM regime reducing the ELM size further. Although usually an improvement of energy confinement is observed using $\mathrm{N}_{2}$ as seeding gas no increased $\mathrm{W}$ concentrations are observed, since obviously the particle confinement is not improved. This is supported by the fact that there is no change of the line averaged density and no signatures of central density peaking. Moreover the sustained regular ELM activity prevents a strong inward transport of $\mathrm{W}$ across the pedestal region [14]. In contrast, when using Ar as the seeding impurity the ELM frequency is strongly reduced. Here the absence of regular flushing of impurities often leads to an increased $c_{W}$, if the ELM frequency is not stabilized by external means. Moreover significant amounts of Ar change also the central particle confinement provoking peaking of the $\mathrm{W}$ density profile. Experiments using gas mixtures have only started, but the deleterious effects accompanying pure Ar are strongly ameliorated, albeit not completely suppressed. An optimisation for different levels of heating power is envisaged to provide a broader data base for the extrapolation to ITER, where the 
use of at least two seeding gases with different radiation characteristics might be necessary to provide a sufficient radiative cooling. 


\section{References}

[1] R. Neu, M. Balden, V. Bobkov, R. Dux, O. Gruber, et al., Plasma Phys. Controlled Fusion 49, B59 (2007).

[2] A. Kallenbach, R. Dux, M. Mayer, R. Neu, T. Pütterich, et al., Nucl. Fusion 49, 045007 (2009).

[3] O. Gruber, A. C. C. Sips, R. Dux, T. Eich, J. C. Fuchs, et al., Nucl. Fusion 49, 115014 (2009).

[4] A. Kallenbach, M. Balden, R. Dux, T. Eich, C. Giroud, et al., submitted to Journ. Nucl. Mater., presented at 19th PSI Conf. San Diego (2010).

[5] R. Neu, R. Dux, A. Geier, A. Kallenbach, R. Pugno, et al., Plasma Phys. Controlled Fusion 44, 811 (2002).

[6] R. Dux, C. Giroud, R. Neu, A. G. Peeters, J. Stober, et al., J. Nucl. Mater. 313-316, 1150 (2003).

[7] A. Kallenbach, R. Dux, J. C. Fuchs, R. Fischer, A. Herrmann, et al., Plasma Phys. Control. Fusion 52, 055002 (2010).

[8] A. Kallenbach, P. T. Lang, R. Dux, J. C. Fuchs, A. Herrmann, et al., J. Nucl. Mater. 337-339, 732 (2005).

[9] K. Schmid, A. Manhard, C. Linsmeier, A. Wiltner, T. Schwarz-Selinger, et al., Nucl. Fusion 50, 025006 (2010).

[10] R. Dux, V. Bobkov, A. Herrmann, A. Janzer, A. Kallenbach, et al., J. Nucl. Mater. 390-391, 858 (2009).

[11] A. Thoma, K. Asmussen, R. Dux, K. Krieger, A. Herrmann, et al., Plasma Phys. Controlled Fusion 39, 1487 (1997).

[12] I. Beigmann, A. Pospieszczyk, G. Sergienko, I. Y. Tolstikhina, and L. Vainshtein, Plasma Phys. Control. Fusion 49, 1833 (2007). 
[13] D. Naujoks, K. Asmussen, M. Bessenrodt-Weberpals, S. Deschka, R. Dux, et al., Nucl. Fusion 36, 671 (1996).

[14] T. Pütterich, R. Dux, A. Janzer, and R. McDermott, submitted to Journ. Nucl. Mater, presented at 19th PSI Conf. San Diego (2010).

[15] T. Pütterich, R. Neu, R. Dux, A. D. Whiteford, M. G. O'Mullane, and ASDEX Upgrade Team, Plasma Phys. Controlled Fusion 50, 085016 (2008).

[16] M. Sertoli, C. Angioni, R. Dux, R. Neu, T. Pütterich, and ASDEX Upgrade Team, subm. to Plasma Phys. Control. Fusion (2010). 


\section{Figure captions}

Fig. 1 . $\mathrm{N}_{2}$ seeded H-Mode discharge \#25394 with $I_{p}=1 \mathrm{MA}, B_{t}=-2.5 \mathrm{~T}$. The $\mathrm{D}_{2}$ puff was kept constant through the main phase of the plasma, whereas the divertor plasma temperature $T_{e}^{d i v}$ was feedback controlled to $8 \mathrm{eV}$. Note the response of the $\mathrm{N}_{2}$ puffing rate to the increase of heating power.

Fig. 2. Averaged W-influx versus a) inter-ELM divertor temperature and b) Nitrogen influx represented by an N IV spectral line in the main chamber. The symbols/colours denote different levels of auxiliary heating power. The data taken at high D puffrate are marked with red circles.

Fig. 3. Peaking of the $\mathrm{W}$ concentration $\left(c_{W}^{\text {centre }} / c_{W}^{\text {edge }}\right.$, top) and edge $\mathrm{W}$ concentration $\left(c_{W}^{\text {edge }}\right.$, bottom) against the $\mathrm{N}$ influx for all discharges under investigation.

Fig. 4. $\mathrm{N}_{2} /$ Ar seeded H-Mode discharge \#25834 with $I_{p}=1 \mathrm{MA}, B_{t}=-2.5 \mathrm{~T}$ similar to that of Fig. 1 but with additional feed forward Ar seeding starting at $2.1 \mathrm{~s}$. As a reaction to the Ar injection the $\mathrm{N}_{2}$ puff is switched off by the feedback control. Note that the Ar puff rate is multiplied by 10 for better illustration. 

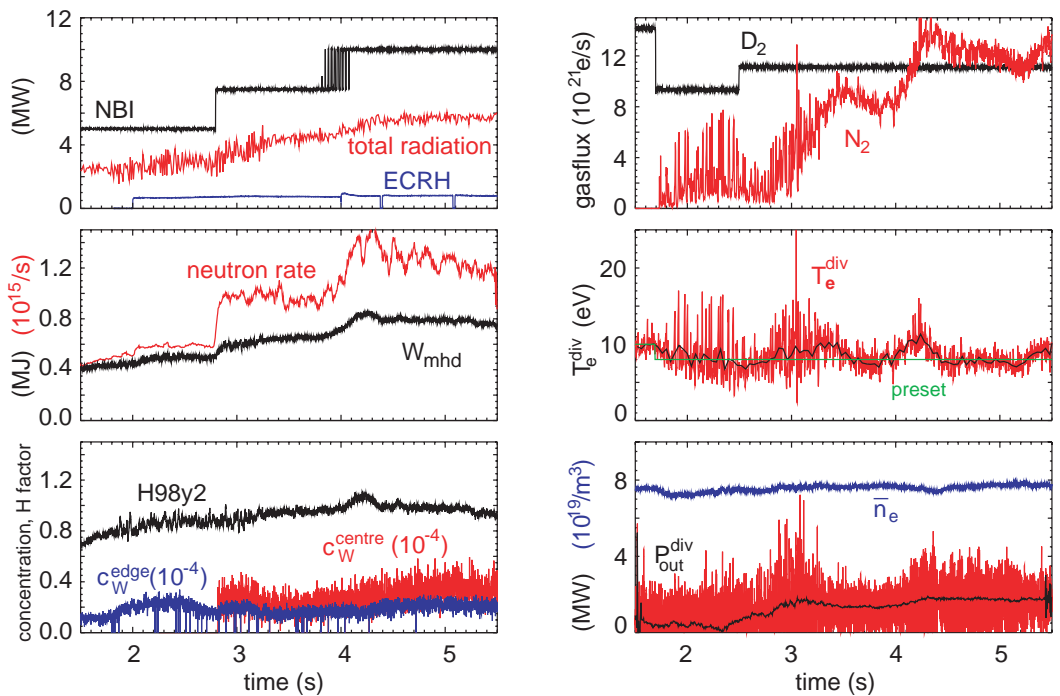

Fig.1 


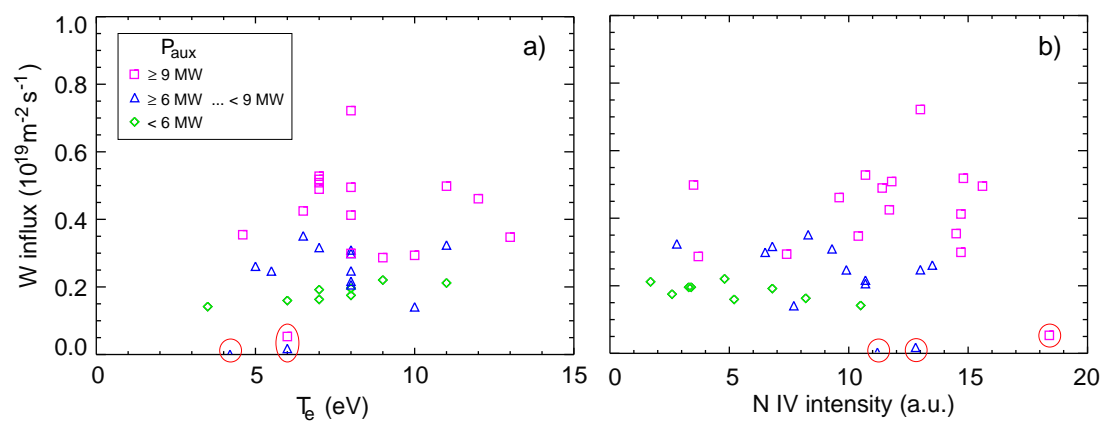

Fig.2 


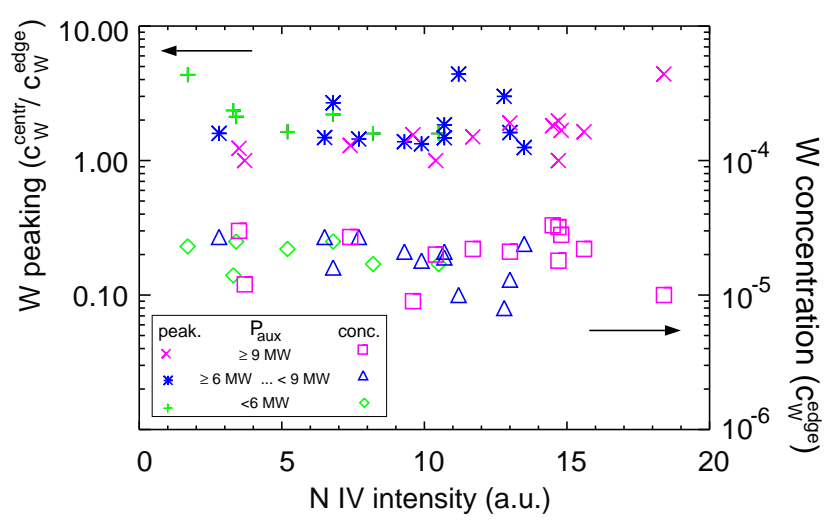

Fig. 3 

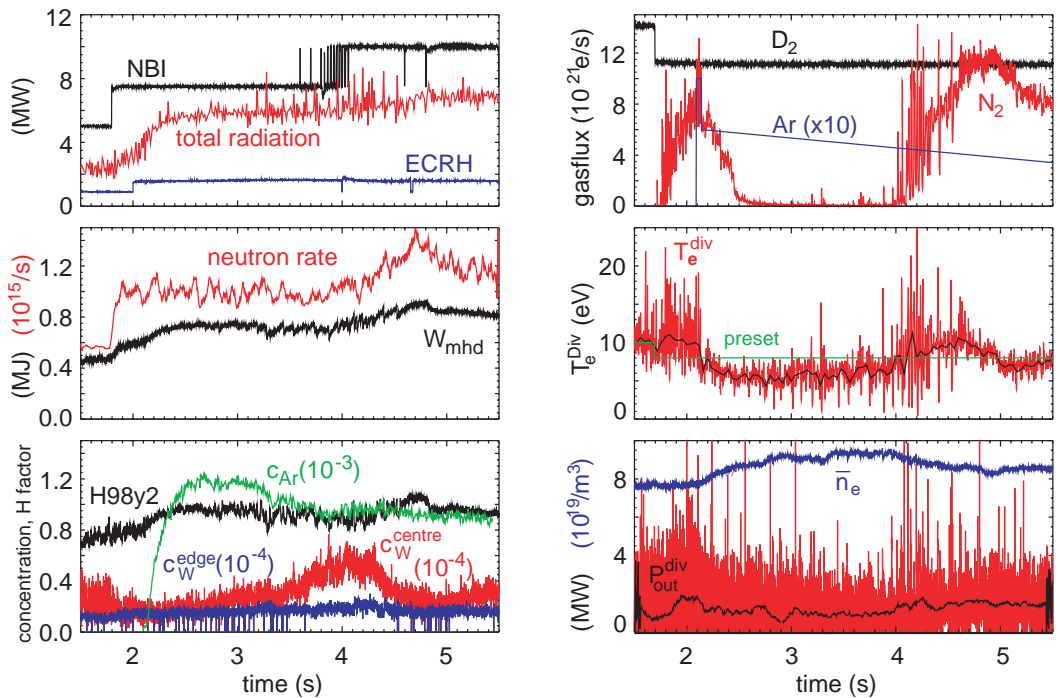

Fig.4 